\title{
NLRP3 polymorphisms and response to interferon-beta in multiple sclerosis patients
}

\author{
Sunny Malhotra, Melissa Sorosina, Jordi Río, Silvia Peroni, Luciana Midaglia, Luisa M Villar, \\ José C Álvarez-Cermeño, Ina Schroeder, Federica Esposito, Ferdinando Clarelli, Uwe K Zettl, \\ Jeannette Lechner-Scott, Nino Spataro, Arcadi Navarro, Giancarlo Comi, Xavier Montalban, \\ Filippo Martinelli-Boneschi and Manuel Comabella
}

\begin{abstract}
We aimed to investigate whether NLR family, pyrin domain containing 3 (NLRP3) polymorphisms are associated with the response to interferon-beta (IFN $\beta$ ) in multiple sclerosis (MS) patients. A total of 14 NLRP3 polymorphisms were genotyped in a cohort of 665 relapsing-remitting MS patients recruited across 5 centers and classified into responders and non-responders according to clinical-radiological criteria after 1 year of IFN $\beta$ treatment. A meta-analysis failed to demonstrate significant associations between the response to IFN $\beta$ and NLRP3 polymorphisms. These findings do not support a role of polymorphisms located in the NLRP3 gene and the response to IFN $\beta$ in MS patients.
\end{abstract}

Keywords: Multiple sclerosis, inflammasome, polymorphisms, interferon-beta

Date received: 19 June 2017; revised: 29 September 2017; accepted: 1 October 2017

\section{Introduction}

Inflammasomes are multi-protein complexes involved in the maturation of proinflammatory cytokines. ${ }^{1}$ One of the best characterized inflammasomes, the NLR family, pyrin domain containing 3 (NLRP3), was recently reported to be associated with the response to interferon-beta (IFN $\beta$ ) in multiple sclerosis (MS) patients, based on the findings of higher NLRP3 messenger RNA expression levels in peripheral blood mononuclear cells from IFN $\beta$ non-responders compared to responders. ${ }^{2}$ The same study showed a trend for association between the NLRP3 polymorphism rs35829419 and the response to IFN $\beta$ according to clinical criteria. ${ }^{2}$ In this study, we genotyped additional NLRP3 polymorphisms in order to further investigate a potential association between the NLRP3 gene and the response to treatment using more stringent clinical-radiological criteria.

\section{Patients and methods}

A total of $665 \mathrm{MS}$ patients were recruited from 5 centers (Milan, $n=389$; Barcelona, $n=107$; Madrid, $n=$ 83; Rostock, $n=68$; Newcastle, $n=18$ ). Inclusion criteria for the study were as follows: (1) age $>18$ years and MS diagnosis according to McDonald crite$\mathrm{ria}^{3}{ }^{3}(2)$ treatment naive patients receiving IFN $\beta$ for at least 6 months and with a follow-up longer than 1 year; (3) relapsing-remitting disease course at the time of treatment onset. Patients who switched between IFN $\beta$ formulations were retained in the study. The study was approved by the corresponding local ethics committees, and written informed consent was obtained from all participants.

Criteria of response to IFN $\beta$ were applied after 1 year of treatment based on clinical-radiological criteria. ${ }^{4}$ Non-responders were patients fulfilling any of the three of the following criteria: (1) presence of relapses, (2) increase of one or more points in the Expanded Disability Status Scale (EDSS) score, and (3) presence of $\geq 3$ active lesions (new or enlarging T2 lesions or contrast-enhancing lesions) on the 1-year brain magnetic resonance imaging (MRI). Patients with no evidence of disease activity according to these variables were considered IFN $\beta$ responders.

\section{Selection of NLRP3 single-nucleotide polymorphisms and genotyping}

Taking advantage of 1000 Genomes Project data, an initial list of variants segregating in CEU (Europeans) population was obtained considering the region
Multiple Sclerosis Journal

$1-4$

DOI: $10.1177 /$

1352458517739137

(C) The Author(s), 2017. Reprints and permissions: http://www.sagepub.co.uk/ journalsPermissions.nav
Correspondence to: M Comabella Servei de NeurologiaNeuroimmunologia, Centre d'Esclerosi Múltiple de Catalunya (Cemcat) and Institut de Recerca Vall d'Hebron (VHIR), Hospital Universitari Vall d'Hebron, Universitat Autònoma de Barcelona, Passeig Vall d'Hebron, 119-129, Barcelona 08035, Spain. manuel.comabella@vhir. org

Sunny Malhotra Jordi Río Luciana Midaglia Xavier Montalban Manuel Comabella Servei de Neurologia-

Neuroimmunologia, Centre d'Esclerosi Múltiple de Catalunya (Cemcat) and Institut de Recerca Vall d'Hebron (VHIR), Hospital Universitari Vall d'Hebron, Universitat Autònoma de Barcelona, Barcelona, Spain

Melissa Sorosina

Silvia Peroni

Ferdinando Clarell Laboratory of Human Genetics of Neurological Disorders, Institute of Experimental Neurology (INSPE), Division of Neuroscience, San Raffaele Scientific Institute, Milan, Italy

Luisa M Villar José C Álvarez-Cermeño Departments of Neurology and Immunology, Hospital Universitario Ramón y Cajal, Instituto Ramón y Cajal de Investigacion Sanitaria, Madrid, Spain

Ina Schroeder Uwe K Zettl Department of Neurology, Neuroimmunological Section, University of Rostock, Rostock, Germany

Federica Esposito Laboratory of Human Genetics of Neurological Disorders, Institute of Experimental Neurology 
(INSPE), Division of

Neuroscience, San Raffaele Scientific Institute, Milan,

Italy/Department of

Neurology, Institute of

Experimental Neurology

(INSPE), Division of

Neuroscience, San Raffaele

Scientific Institute, Milan,

Italy

Jeannette Lechner-Scott Department of Neurology, John Hunter Hospital,

Newcastle, NSW, Australia/ Hunter Medical Research

Institute, The University of

Newcastle, Newcastle, NSW, Australia

Nino Spataro

Centre for Genomic

Regulation (CRG), The

Barcelona Institute of Science and Technology, Barcelona,

Spain/Universitat Pompeu

Fabra (UPF), Barcelona,

Spain

Arcadi Navarro

National Institute for

Bioinformatics, Universitat

Pompeu Fabra, Barcelona,

Spain/Institució Catalana de

Recerca i Estudis Avançats

(ICREA), Barcelona, Spain

Giancarlo Comi

Department of Neurology,

Institute of Experimental

Neurology (INSPE), Division

of Neuroscience, San

Raffaele Scientific Institute,

Milan, Italy

Filippo Martinelli-Boneschi

Laboratory of Genetics of

Complex Disorders and

Department of Neurology,

IRCCS Policlinico San

Donato, Milan, Italy/

Department of Biomedical

Sciences for Health,

University of Milan, Milan, Italy

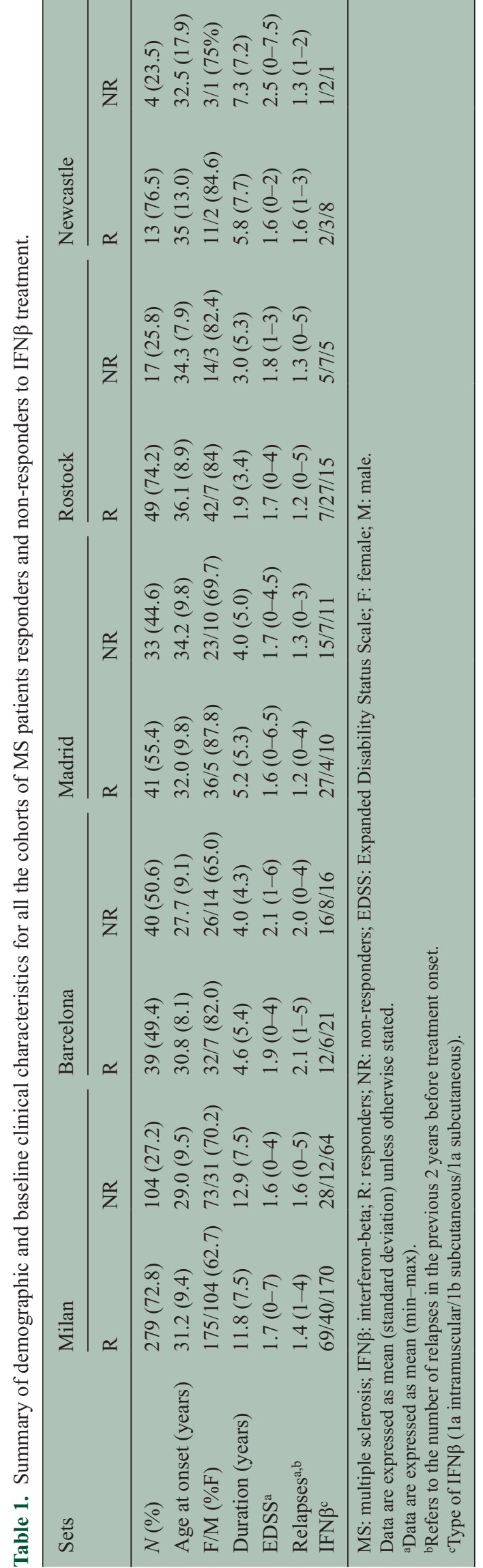

spanning $5 \mathrm{~Kb}$ NLRP3 gene start and gene end. Among all the obtained variants, only 26 were found to be annotated in at least one of the several used functional and conservation databases. Finally, only 14 variants had a valid TaqMan code and were considered for further analysis.

Genotyping was conducted using a TaqMan Open Array Genotyping System (Thermo Fisher Scientific, Waltham, USA). DNA samples were loaded at $50 \mathrm{ng} /$ $\mathrm{mL}$ and amplified according to the manufacturer's instructions. The autocalling method as implemented in the TaqMan Genotyper software version 1.3 was used to assign genotypes. As quality controls, a genotype call rate of 0.90 and an individual call rate of 0.80 were used as threshold.

\section{Statistical analyses}

Gender, age at onset, disease duration, number of relapses in the 2 years before treatment onset, and baseline EDSS were tested as possible confounding variables within each cohort using the $t$-test or MannWhitney test when appropriate for continuous data and the chi-square test for ordinal data and comparing responders and non-responders. All single-nucleotide polymorphisms (SNPs) were tested for association with response using PLINK-v1.07. ${ }^{5}$ Statistical significance of individual genotype data was calculated using a logistic regression model within each cohort adjusted for any clinical variable identified as differentially distributed across responders and nonresponders. Odds ratios (ORs) and 95\% confidence intervals (CIs) are reported. PLINK-v1.07 was also used for evidence of deviation from Hardy-Weinberg equilibrium within each cohort. A meta-analysis across the cohorts was performed assuming a perallelic model and using both fixed- and randomeffects meta-analysis approaches. ${ }^{6}$

\section{Results}

A total of 619 patients were included in the analysis while 46 patients were excluded due to low call rate. A total of 421 patients $(68.0 \%)$ were classified as responders and 198 patients $(32.0 \%)$ as non-responders. Table 1 summarizes the main demographic and clinical characteristics of patients. No differences were observed between responders and non-responders except for the number of relapses in the 2 years before treatment onset in the Milan cohort $(p=0.03)$; thus, this variable was included as covariate in the analysis.

Among the 14 selected NLRP3 polymorphisms, the following were excluded: (1) rs4925547 and 


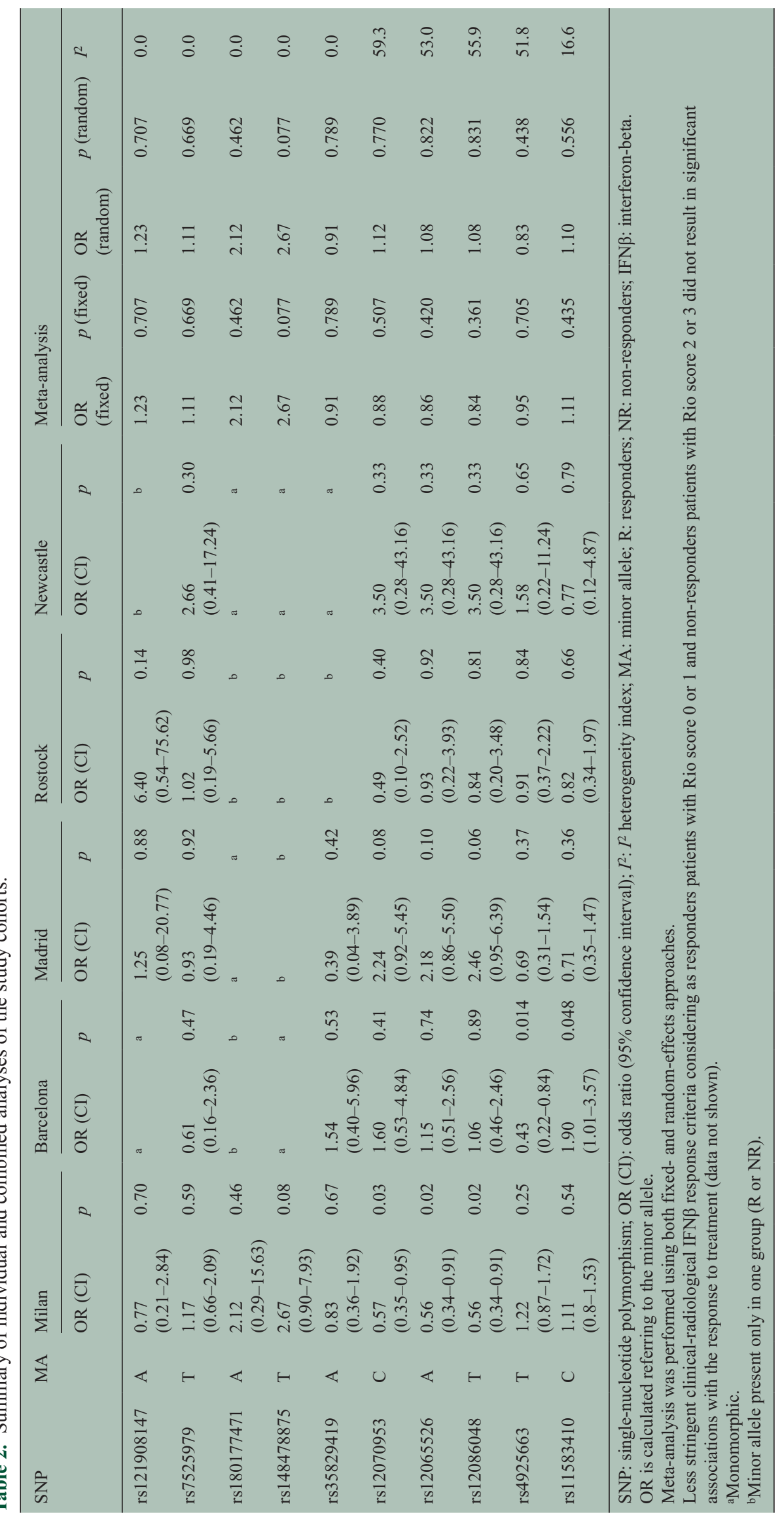


rs34298354 had a call rate $<0.90$, (2) rs201229629 was monomorphic in the entire cohort, and (3) rs139814109-T allele was not present in the nonresponder cohort, thus it was not possible to calculate an association. A final list of 10 SNPs was included in the analyses. None of them showed deviation from Hardy-Weinberg equilibrium $(p>0.05)$.

As shown in Table 2, individual cohort analysis only revealed marginal associations with the response to IFN $\beta$ for the NLRP3 polymorphisms rs12070953, rs12065526, and rs12086048 in the Italian cohort and for rs4925663 and rs11583410 in the Barcelona cohort. However, in the meta-analysis, none of the SNPs genotyped were significantly associated with the response to IFN $\beta$. A trend for association was observed in the meta-analysis for SNP rs148478875; however, this effect was exclusively driven by the results in the Italian cohort, since the polymorphism was found to be monomorphic in the other cohorts most likely due to the low frequency of the minor allele.

\section{Discussion}

In an earlier study, ${ }^{2}$ the NLRP3 polymorphism rs35829419 showed a trend for association with the response to IFN $\beta$ in MS patients classified into responders and non-responder according to clinical criteria based on the presence of relapses and progression on the EDSS score after 2 years of treatment. Motivated by these findings, in this study, we selected additional SNPs across the NLRP3 gene in order to investigate a relationship between inflammasome polymorphisms and response to treatment in MS patients classified into responders and non-responders according to more appropriate stringent clinicalradiological criteria. ${ }^{4}$ Combined analysis of the five MS cohorts did not show a significant association between rs35829419 and IFN $\beta$ response, and genotyping of additional NLRP3 polymorphisms also failed to demonstrate significant associations with

treatment response. Altogether, these results do not support a role for NLRP3 polymorphisms and IFN $\beta$ response in MS patients.

\section{Acknowledgements}

S.M. and M.S. contributed equally to this work.

\section{Declaration of Conflicting Interests}

The author(s) declared no potential conflicts of interest with respect to the research, authorship, and/or publication of this article.

\section{Funding}

The author(s) disclosed receipt of the following financial support for the research, authorship, and/or publication of this article: This work was supported by a grant from the "Fondo de Investigación Sanitaria" (FIS; grant number PI16/00924), Ministry of Science and Innovation, Spain.

\section{References}

1. Guarda G, Braun M, Staehli F, et al. Type I interferon inhibits interleukin-1 production and inflammasome activation. Immunity 2011; 34: 213-223.

2. Malhotra S, Río J, Urcelay E, et al. NLRP3 inflammasome is associated with the response to IFN- $\beta$ in patients with multiple sclerosis. Brain 2015; 138(Pt3): 644-652.

3. Polman CH, Reingold SC, Banwell B, et al. Diagnostic criteria for multiple sclerosis: 2010 revisions to the McDonald criteria. Ann Neurol 2011; 69: 292-302.

4. Río J, Castilló J, Rovira A, et al. Measures in the first year of therapy predict the response to interferon beta in MS. Mult Scler 2009; 15: 848-853.

5. Purcell S, Neale B, Todd-Brown K, et al. PLINK: A tool set for whole-genome association and populationbased linkage analyses. Am J Hum Genet 2007; 81: $559-575$.

6. Mantel N and Haenszel W. Statistical aspects of the analysis of data from retrospective studies of disease. J Natl Cancer Inst 1959; 22: 719-748. journals.sagepub.com/ home/msj

๑) SAGE journals 